\title{
Improving multidisciplinary communication at ward board rounds using video enhanced reflective practice
}

\author{
Cyril Hellier, Vicki Tully, Sandra Forrest, Pamela Jaggard, Morag MacRae, Dirk Habicht, Alexandra Greene, Karen Collins \\ Tayside Patient Safety UK
}

\begin{abstract}
The priority to ensure patient safety and use resources effectively, demands attention and innovation. Video enhanced reflective practice (VERP) provides training based upon analysis of film clips of one's professional practice to develop practical insight into the processes of communication, so that effective changes can be made to ongoing behaviour and practice.
\end{abstract}

In this case the focus was on multi-disciplinary communication within daily board rounds on an acute medicine and care of the elderly ward. Baseline assessment and post intervention testing of perceptions of change by both full and core team were undertaken to establish the impact of VERP training. In addition pre and post focus group discussion and film analysis supplemented evaluation.

The findings support the view that after VERP training of a core team, board rounds were seen as consistently easier to participate in, providing improved focus, were more efficient in goal setting and resulting in better care for patients as well as improved pathways to discharge. This suggests benefits to the communication "culture" of a multidisciplinary team resulting in increased benefits for the wider team. It is concluded that the use of tailored VERP training for personal, professional and team development is relevant, feasible, and worthy of further testing and investigation.

\section{Problem}

A board round is a process which should improve communication among the multi-disciplinary team (MDT), enhancing team working and providing a more coordinated approach to discharge planning. In our pilot ward, an acute medical and care of the elderly ward, board rounds had been in place for around three years. For the board round all team members are expected to assemble at 11.10 am each morning in front of the white board at the nurses station. This board contains patient details including planned date of discharge, any risk factors to patient safety and input from physiotherapy,occupational therapy or social work. The board rounds are attended by the nurse in charge, consultant, middle grade and junior doctors, junior and senior nurses, social worker, physiotherapist, occupational therapist and ward clerk among others. There follows a twenty minute discussion of the thirty inpatients in the ward with an emphasis on facilitating early and safe discharge. This process requires efficient communication of all and input from different members of the team depending on the case discussed. An inefficient board round process leads to delays in discharging patients. In our pilot ward it was recognised that board rounds were often not as efficient and useful as they could be.

Rarely was it possible to contain the discussion to its allocated time of twenty minutes, and there were frequent distractions and often not all staff were present. Some improvement work had already been undertaken as part of a breakthrough series improvement collaborative (OPAC) in NHS Tayside which set out to improve the experience of older people in our acute care hospital wards. Several PDSA (Plan, Do, Study, Act) cycles were undertaken to improve the process and included testing moving the board round to the staff room to avoid distractions. However, none of the tests resulted in improvement, so frustration with the process grew. During board rounds medical staff tended to be dominant without contributing enough relevant information, such as the functional status of a patient and social care needs. Junior nursing staff often did not contribute despite possessing the relevant information on the functional ability of a patient. Due to a lack of the appropriate information available at the board round, conclusions could often not be reached and decisions about patients were often deferred.

In a wider context, the importance of effective communication within MDT's is recognised. The World Health Organisation [5] highlighted the importance of human factors in patient safety by identifying the key features of communication and teamwork. It is also worth noting the recommendations from the Francis Inquiry [6] which highlighted the importance of communication skills and ward cultures as features of professional quality and performance in delivering health services as "collective care". Indeed recommendation number 237 states, "There needs to be effective teamwork between all the different disciplines and services that together provide the collective care often required by an elderly patient."

\section{Background}

Without practical tools and concrete exemplars it is hard to know if positive culture and effective teamwork are present as a means to delivering the ends of successful patent experience and outcomes.

Key questions arise as to how we change culture and evidence improvement. Staff collaboration in planning and delivery of patient 
care and treatment depends on individual and collective processes that are fundamentally premised on effective communication. Communication is clearly a central element, but as a non technical skill is not often the direct focus in the training of nursing, support, and medical staff within the NHS.

Without evaluation and detailed analysis (as can be performed easily on a piece of film) it is hard to know if positive culture and effective teamwork are present as a means to delivering the ends of successful patient experience and outcomes.

Developing tailored video enhanced reflective practice (VERP) for multi-disciplinary team (MDT) planning processes within the NHS is untried, yet seems highly relevant to widespread stated needs for improvement. VERP is an empowering and strengths based approach seeking to improve communication, reflections and critical analysis. Application to professional settings and relationships is relatively recent and is at the stage where new contexts will require tailor made input, reflecting on actual work settings. It derives from video interaction guidance (VIG) [8], which has been developed in the past fifteen years, initially introduced to Scotland, and then to the rest of the UK and is now used in many European countries.

Video interaction guidance has built up a significant evidence base to demonstrate relevance to diverse client groups indicating that it is methodologically sound and based upon a solid theory base. Its aim is to develop practical insight into the processes of communication by using short clips of video to help professionals reflect on when communication is going well so that changes can be made to ongoing practice that will assist in positive change and improvement. Insight develops directly from reviewing one's personal behaviour in the actual situation (on film) in which change is required. Its potential application to improve safety is wide, from parenting through to a host of professional working settings where communication is central to outcomes. Publications $[9,10]$ highlight burgeoning application. Hellier [11] and Greene [12], 'Integrating VERP into Medical Education, In Video Enhanced Reflective Practice. Professional development through attuned interactions' (in press April 2015) demonstrates applicability of spreading training to new organisations, including careers advisory services and further education in Scotland.

The aim is to build insights about one's communication that empower and inform one's behaviour. How one contributes, facilitates the contribution of others, or ensures full discussion in agreeing decisions (eg about patient treatment plans or discharge) becomes the focus for discussion within training, which is conducted in a constructive and positive way throughout. Training can be undertaken by an individual or small group/team, something that is negotiated at the outset. In this pilot it was anticipated that core team-building would be a by-product of training together, along with shared problem-solving of issues arising during board rounds.

The team first heard about video interactive guidance during a learning session for the older people in acute care collaborative (OPAC) and became interested in the concept. Multidisciplinary team communication was a key workstream of the collaborative and a presentation was delivered by Dr Alexandra Greene, senior research fellow in medical anthropology at Dundee University focusing on effective communication and "tribal" behaviour within the NHS. It was agreed to support a test of change using VERP within this one ward supported by the NHS Tayside patient safety team.

\section{Baseline measurement}

A baseline survey questionnaire was constructed and administered prior to the intervention with the purpose of sampling the views of the multidisciplinary team participants in the daily board round meetings. This had to be a sample, given the nature of the team; a large and changing membership, dependent on timing (turnover of eg junior doctors), and time of day (changing shifts of nursing staff).

Questions aimed to establish the role of the professional within the team and length of time having participated at board rounds, their views of its accessibility, and its contribution to improving patient outcomes. Views were also sought as to the strengths and needs for further development of the board round process. In addition there was a baseline element focused upon personal communication skills in professional communication and perceived needs for development. A solution focus and appreciative inquiry approach underpinned the construction of the survey tool. The initial survey completed in May/June 2014 was necessary to build a picture of the wider context in which the VERP training of the core team was to take place.

Additionally a pre-intervention focus group with the core team was held in June 2014 to discuss responses to the questions in the initial survey in more detail. The initial survey was useful to build a picture of the wider context in which the VERP training of four core team members was to take place.

In addition, three focus groups were organised: the pre-intervention focus group was held with the core team in June 2014 to discuss the aims for the pilot, as well as providing an opportunity to gather in-depth responses to the questions in the initial survey; a postfocus group with the core team gathered data of the teams experiences of having completed the VERP sessions, and a postfocus group with the wider multidisciplinary team explored attitudes towards the board round process pre-and post intervention.

See supplementary file: ds4584.doc - "Board Round Questionairre Final April 2014"

\section{Design}

Video enhanced reflective practice (VERP) training was undertaken with four members of the MDT (a lead consultant, senior charge nurse, occupational therapist, and physiotherapist) of our test ward, this formed the core team. This involved the delivery of six sessions in July and August 2014, each based upon analysis of a new film of a board round. Aims included to:

- Promote a more co-ordinated and timely approach to a patient's hospital journey 
- Encourage members of the MDT to contribute to the board rounds

- Support multidisciplinary goals to be set for on-going patient care

- Consider the long-term sustainability of this intervention in a resource-limited environment, ultimately determining its suitability for further use in other units in the NHS.

What was innovative was the filming and analysis of board rounds by team members, facilitated by a psychologist and VIG-trainer allowing for the consideration of individual contributions as well as effectiveness as a team. It also offered training and development in situ on the ward, requiring relatively little time away from delivery of patient care and from direct working in a MDT. It was conducted as action research, allowing for the evaluation of impact on team members. Filming board round meetings over a two month period required a careful analysis of each film, in order to structure discussion during training sessions.

The attunement principles provide a framework to identify patterns and elements of communication. These simple-to-apply principles were used to reflect on the filmed communication skills and behaviours highlighting issues relating to professional communication and identifying patterns in eg role of contributors, length of time speaking, and time allocated to each patient discussed. This allowed for comparison of patterns in early and later films, to chart any change. It was decided to make a postintervention film for analysis in this way, once the training had been completed. By definition, such information provided data for further analysis and its potential use is discussed later in lessons and limitations.

This application of attunement principles has worked well in, for example, improving parenting skills, parent-child relationships, or teaching skills and student-teacher relationships. These principles are organised into four blocks to ease access and application, each providing a structure to consider features of individual and group interaction. It was necessary to adapt the blocks with questions specific to the NHS context.

This structure allowed reflection on examples and patterns of body language, aspects of sharing information as well as sophisticated aspects of co-operation and collaboration, all relative to the goals and purposes of the board round process. In effect it made clear the communication processes that underlie the "culture" of a group and allowed participants to consider their choices in communication behaviour in the future.

To enhance sustainability, a periodic review of team working through incidental filming and analysis of ongoing board rounds (perhaps every six months) was raised as a way of ensuring adherence to the attunement principles and continuous improvement in team communication.

\section{Strategy}

The VERP pilot was structured on "action research" principles and although this does not neatly fit with the traditional PDSA cycles we have used our data and learning to inform the improvement process.

Each video analysis session provided the core team members with new communication and leadership skills that were then applied in the daily board rounds.

PDSA cycle 1: The pilot set out to address key questions, including: can "culture" and "teamwork" be made accessible to training and development in the context of delivering safe care? Would it be possible to improve teamwork through focus on communication at daily board rounds, with the aim of making patient planning more effective? Having identified VERP as a potentially relevant methodology for reflection and analysis, could a pilot study be developed to demonstrate its accessibility and feasibility with its aim to improve professional collaboration? Given that staff would find it difficult to take significant time away from direct working with patients, would training based in the working environment prove effective and be acceptable?

Data collection: what information could be gathered that draws on the perceptions of team members and allows baseline and post training feedback? Given the extremely busy nature of such work it was decided to supplement any questionnaire findings with pre and post focus group interviews to allow more in depth discussion. The use of film analysis for training was anticipated to provide another source of information as an indicator of positive change. Impact on both the trained core team and the non trained wider team would allow a degree of cross checking; also asking new team members about the ease of participation and value of board rounds would provide different subgroups of respondents.

If successful, the training was anticipated to team-build within the core team, impacting positively on the process of managing board rounds and be seen by them to add value to planning and treatment of patients, ultimately contributing to a more streamlined discharge pathway. Ideally any movement in these directions would need to be recognised within the wider team.

PDSA cycle 2: Training was developed for the core team and was originally envisaged to last for six hourly sessions, but in the event that it coincided with a core team member's holiday period, another session was provided for catch up purposes. In addition, core members were offered one extra personal session for those wishing to focus on individual communication styles, and this was taken up by two members of the core team. In practice, most sessions had all four core team members present and it was possible to use peer reflection to support individual and group learning.

Each session focused upon the most recent board round that had been filmed and was usually within a week to ten days of the filming. This ensured that participants could remember details of each particular board round.

PDSA cycle 3: The training, based upon use of the attunement principles as a framework, clearly helped the core team to see repeated patterns in participation and responses of team members in board rounds. It led to lively discussion as to how to correct a 
perceived imbalance in participation, for example the relative domination by medical staff and a relative dearth of nursing input in initial sessions. Positive alliances, eg between physio and occupational therapy were evident, as was the value of particular questions to promote discussion, build consensus, and be clear on agreed outcomes. The value of being prepared for board round discussion, having the relevant breadth of expertise and keeping to time were seen as critical to a successful board round.

The training process of working on individual communication within a shared board round, building the core team as a collegiate group, allowed for peer support through identification and reinforcement of successful strategies. Also the use of self and peer modelling of communication behaviours, eg active listening to colleagues or use of humour to share group responses to an issue.

PDSA cycle 4: Following evaluation of the VERP training which was tailored to suit the context of team planning for patients, it was apparent that the group process adopted was suitable to delivery of personal and core team development. This methodology is now available to streamline and adapt to other team settings within Tayside. Key learning points include improved understanding of how members of a core team can actively facilitate full team participation and ensure efficient decision making at board rounds. One example to illustrate this is the realisation that well formed questions are critical to summarising, checking for shared understanding and achieving consensus around decisions regarding treatment and discharge date for a particular patient. A review of all filmed sessions will allow for categorisation and collation of examples of different questions for the development of future training materials. Furthermore, the selection of good examples of recorded (filmed) interaction, such as an information exchange between medical, nursing, therapeutic, or social work representatives, will be possible for future sharing of practice across teams.

It is intended to further refine the training process building on the test experience. Assessment results suggested that the six sessions were adequate for the stated aims, although due to holiday leave and offers of 'catch up' training to individuals, it was apparent the opportunity to have an offer of one personal session could well be built into future refinement of a training model.

See supplementary file: ds4575.doc - "PDSA cycles"

\section{Post-measurement}

Pre-training: Qualitative data was collected (questionnaires) and returns from the initial full team survey were completed in June/July 2014 by seventeen respondents. These covered a wide range of roles including consultant, junior doctor, senior charge nurse, charge nurse, student nurse, physiotherapist, occupational therapist, psychiatric nurse, patient flow coordinator, dietician, and social worker. The majority reported that ease of participation was relatively high. A third of comments on participation focused upon the confidence that respondents felt when taking part eg, "I feel confident in my role and it is a good forum to participate." The way the board rounds operated was seen to facilitate participation,
"Each member of the MDT has time to give their update on each patient."

When asked about the most successful aspects of board rounds, the responses highlighted the value of the process as well as their impact on planning. They were clearly appreciated as providing a guaranteed opportunity to discuss patients that was managed and frequent. The coordinating aspect for MDT working was a repeated theme, emphasising the development of professional relationships. Significant benefits for patients were identified, and these included the facilitation of decision making in a complex environment where many different services are required to collaborate. The benefits to the planning process were seen to include the engagement of a wide range of professions and the maximisation of resources.

Continuing challenges included ensuring that all relevant team members were prepared and did attend on time for discussion, and that board rounds were well managed and used limited time effectively. There was also a need to get all players on board and participating well and to improve record keeping arising from discussion and agreement at board rounds. How to ensure follow up action was identified as an ongoing issue.

Post-training questions focused on the impact of VERP on personal and core team communication knowledge and skills, and on any perceived changes in MDT functioning, ultimately to impact on patient experience. This questionnaire was completed in August 2014.

Following the VERP training sessions, an evaluation of impact reported by the core team was extremely positive. At the outset each participant identified individual goals in undertaking communication training; all four core team members reported positive gains in these as a result of VERP for both personal goals in improving communication skills as well as working effectively as a team. With regard to communication, all four core team participants rated themselves relatively highly at the outset on communication in board rounds prior to training, but still reported an increase in professional communication skills after training (from an average of 8.3 to 9.5 on a ten point scale). An increased confidence and self awareness of strengths in communication was evident for each member of the core team, albeit different aspects being acknowledged. Greater self awareness of communication (including body language) in fulfilling a role, eg leading discussion, asserting a point, summarising, and engaging colleagues in discussion were cited, all helping to build the shared responsibility as a core team to maximise board rounds. Knowledge gained included a more sophisticated understanding of communication strategies, as well as the use of a new focus on the dynamic of a complex group process.

Each core team member reported an impact on the functioning of the MDT as a result of their personal developments. Improvements in the atmosphere of the team and increases in relevance of information shared were reported. Specific groups who were originally of concern, eg nursing staff were now seen to be "more freely contributing and being asked to contribute", or junior doctors who were subsequently "giving more appropriate information in 
response to more specific questions." There was also a consistent view reported that there were improvements in supporting MDT goal setting for ongoing patient care, a topic that frequently arose during video reflection and consequent discussion in the training. For example, "with the improved input of information from more members of the wider team we have more accurate and realistic discharge plans."

On the subject of contribution to a more co-ordinated and timely patient experience, core team members recognised the complexities involved in isolating this key outcome, but all saw VERP training as adding value in this respect. The improved focus and better facilitation of discussion were highlighted as key factors; one possible explanation was, "The patient plan is now the main focus." As a result there was seen to be an improved focus on forward goals that, "are more co-ordinated and timely as the whole team is aware of what we are trying to achieve for each individual patient."

Changes reported by the core team were supported by feedback from members of the wider MDT who had been involved in board rounds long enough to confirm either way, but whom had received no direct training. Thirteen of them completed a follow up questionnaire. This "longstanding group" was an exceptionally well experienced group (averaging 2.5 years in this MDT), comprised mainly of nursing staff of all grades (included were a patient flow coordinator, a psychiatry liaison nurse, one consultant, and one ward assistant). The consultant and therapy members of the core team did not complete this questionnaire having already undertaken separate evaluation (focus group). In addition, responses were gained from a further six members who had recently joined the team and were unable to comment on changes over time. Their average length of experience was six weeks, with a range of one week to two months; this illustrates the degree of turnover in, and the wide range of, staff. Four of this subgroup were junior doctors (FY1s) and two were nursing staff (including one student). The feedback from this "new members group" provided an additional perspective on the culture and experience of board rounds on this particular ward.

The vast majority (92\%) of the longstanding group reported noticing some positive changes in the board rounds in the previous three months. These could be grouped into features relating to the management process, the time taken and the perceived efficiency of the meetings. Having an improved structure, whereby medical staff usually led discussion on each patient, was seen to be positive by one third of respondents. Most reported improvements in the speed and efficiency in which board rounds were managed, "more focused", "more efficient", "nurses leading the rounds are more timely". In addition, half reported improvements in attendance, participation, and the range of input to MDT discussion, "the whole team now participates."

The majority (69\%) of the longstanding group reported changes that were seen to have a positive impact on outcomes for patients. Some of these related to the perceived operational improvement reported above, eg the fact that participation of the full team contributed to improved patient outcomes. One noted that discussion was "more patient centred" and two others highlighted the value for patient continuity now that medical staff were better coordinated, "co-ordination of patients overseen by ward doctors not just, say the care team." Most identified improvements in specificity and effectiveness of discharge planning for patients resulting from improved communication and collaboration, "quicker medical plan therefore quicker discharge date." One third highlighted changes in ensuring that different agencies, including therapies, nursing and social work were tasked and engaged.

Overall the full team follow up findings supported those of the baseline survey about the perceived value of board rounds to provide an opportunity to share information and prioritise clinical workload, to the benefit of patients. Findings were positive and supported the views of core team members that post VERP training; board rounds were seen as consistently easier to participate in, providing improved focus and efficiency, resulting in better care for patients as well as improved pathways to discharge.

Film analysis pre and post intervention (VERP) allowed identification of both total length of time taken to contribute by each member as well as how many times they spoke (number of "turns"). Initially the medical contribution represented nearly half the total time (44\%), taking over one quarter of all turns $(26 \%)$; this reduced significantly by nearly half to approximately one quarter of total time (26\%), also taking less turns (22\%). The data suggest that medical contributions shifted to become more concise with average length of turns reducing from 8.6 seconds to 6.7 seconds.

Nursing contribution showed a corresponding improvement initially making up only $5 \%$ of total input and taking less than one in ten turns $(9 \%)$; at follow up this had shifted significantly by a factor of four ( $19 \%$ of total time) and taking one third more turns ( $12 \%$ of total turns). The data suggests that nurses were taking significantly longer turns, more than doubling their average length of turn from three to seven seconds. Post VERP training, their average length of turn equated with that of medical participants, evidencing an empowerment of these members of the MDT.

In addition the total time of the post intervention board round in comparison to the baseline, had reduced by over three minutes (13\%) from 25 minutes 18 seconds to 21 minutes 54 seconds, suggesting a more efficient process.

Finally, a post focus group comprising mainly nursing staff provided further details in supporting the findings that improvements were noted by wider team members who had not been trained.

See supplementary file: ds4576.doc - "Questionniare results"

\section{Lessons and limitations}

With regard to acceptability, the question arises as to how VERP fits with previous training and experience. NHS colleagues do not necessarily get direct training in such "non technical skills" as communication and make little, if any, use of video for review of personal behaviour and working in a team. For example, parallel work in Tayside using VERP support with medical students 
BMJ Quality Improvement Reports

highlighted anxiety for some when having to make one recording of communication with a patient where they had to impart difficult news regarding a diagnosis.

Individual predisposition to training that involves video recording will include uncertainty as to what is expected of them, with some initial anxiety about being filmed and/or a degree of scepticism. To some extent this normal reaction was the case for all those trained in this pilot, but all adjusted quickly (by session two) to seeing the potential value of such personal scrutiny in a working context and all concluded that the new experience of self review was extremely powerful.

With regard to feasibility, the challenge of taking time away from direct working was always present, but in this pilot the use of six one hour sessions over lunchtime worked well in practice. There were two occasions when a bleep was briefly responded to, but in the main the participants were able to focus and work well both as individual learners and as a group. The group methodology was seen to add value both by way of reinforcing personal learning, witnessed in parallel with colleagues, but also by building core team identity through shared learning and shared focus on joint problem solving of issues arising.

The issue of seeking individual written permissions for video recording of large groups (up to twenty) in each board round did represent an administrative burden. This initially required the chasing up of those who had not completed a form, when they were to be on film for the first time. However, this was eventually streamlined using a standardised group form (consistent with Tayside policy on filming) adapted for the purpose.

As for evaluation, more thought on harder data, evaluating impact on wider variables of patient outcome is required; one will have to be realistic about the contribution of one input (VERP) to a chain of causal variables that will all impact on outcomes. Future consideration about the evaluation of hard data including complaints and patient satisfaction and average length of stay is required to reinforce such findings. Certainly VERP does contribute to essential aspects of communication, culture, and teamwork.

Considering the relevance of further dissemination of VERP training to colleagues in other multi disciplinary settings, there was a shared view that it is well suited to a range of settings and adding value, helping to identify areas for improvement as well as charting positive changes.

At this stage of innovating a new and creative solution to the challenges faced in delivering patient safety, VERP training does require highly trained / skilled trainer(s) well versed in VIG / VERP. Working on individual communication skills in a small group setting requires negotiation at the outset and awareness of sensitivities potentially involved. It also requires flexibility in adapting to a new context. Ideally the aim is to build a sustainable model of in-house training that will be fed by ongoing training and developments and use local examples of good practice for illustration. Use of the Tayside patient safety network will ensure that practice is shared and compared.

\section{Conclusion}

Given the flexibility of VERP as a methodology, the decision to focus in this instance on training the core team with the aim to impact on the fuller team, does appear to be justified by the results. Any MDT in such circumstances comprises a wide range and a large number of professional staff, who are subject to high levels of turnover, with junior doctors changing frequently and nursing staff changing shifts regularly. Adding the impact of such changes within professional disciplines and the factor of holiday leave makes it hard to see how one could ever train all members of a team. This pilot evidences the value of training a relatively stable core team that can co-work to facilitate and manage change over time. However, there was a risk in that there was no guarantee that any perceived improvement by those directly trained, would be acknowledged by others who were indirectly involved. In light of the results, it seems appropriate to consider any future training using this model and to continue evaluation to fine-tune delivery.

The Tayside experience of using board rounds to co-ordinate MDT planning for patients was clearly seen as beneficial throughout this study, even in baseline evaluation. This is perhaps not surprising as the team involved, was in some ways self selected in pursuit of building on good practice. However, the input of six hours specialised training in video enhanced reflective practice to the core team, was seen to have significant positive effects on personal learning and shared practice as facilitators of the wider team at board rounds. As a result, discernible benefits to the process and outcomes of the MDT were reported by both core and full team members, in the interests of patient safety.

The methodology, which was tailored to this NHS context using a facilitated group experience and context specific questions, appears to suit a MDT setting well and to have been positively received. In addition, the training stimulated thinking about practice change in management of participation as well as recording outcomes of board round discussion, with greater emphasis on accountability and follow up of proposed action. External confirmation that the board round was working effectively was provided by an unannounced inspection by Health Improvement Scotland [13], who identified it as an example of good practice near the end of the training period.

These findings represent the MDT's perceptions of the VERP sessions and their effect on the board round process, with participants believing that board rounds were consistently easier to participate in (also supported by new members), provided improved focus, were more efficient, and resulted in better care for patients, as well as providing improved pathways to discharge. Applying VERP to the complexities of MDT working appears to have definite relevance to important quality issues relating to communication, collaboration, and culture, all with the common aims of improving continuity and outcomes for patients. This shared view on the benefits to co-operation and co-working in one NHS setting, appeared to be present across team members / different disciplines. Further research and development is required to explore the effects these board round changes have on patient outcomes. 
BMJ Quality Improvement Reports

It is concluded that the use of VERP for individual and team development is worthy of testing and investigation while giving consideration of its long-term sustainability as an intervention in a resource-limited environment. It is further concluded that development of a measure of team interaction and effective communication derived from video analysis should be further developed to apply in any future training. The value of such input needs to be viewed in the context of an imperative to maximise precious resources that impact on patient safety, at a time when achieving best value is paramount.

\section{References}

1. Department of Health Independent inquiry into care provided by Mid Staffordshire NHS

Foundation Trust January 2005-March 2009. Chaired by Robert Francis. HC 375-1. (2010)

Stationery Office.

1. Department of Health Report of the Mid Staffordshire Foundation NHS Trust Public Inquiry

Volumes 1-3. HC-898-I-III. (2013) Stationary Office.

1. Alzheimer's Society Counting the cost: Caring for people with dementia on hospital wards, Alzheimer's Society: (2009) London.

2. Pinner G, Hillam J, Branton T. and Ramakrishnan A. Faculty report FR/OA/1. In-patient care for older people within mental health services. Available at:

https://www.rcpsych.ac.uk/pdf/FR_OA_1_forweb.pdf (accessed on 4.11.2014).

1. Methods and measures Working Group of WHO patient safety. 2009, Human Factors in Patient Safety Review of Topics and Tools [pdf] WHO. Available at:

http://www.who.int/patientsafety/research/methods_measures/huma n factors/human factors review.pdf (Accessed on 0511 2014).

1. The Mid Staffordshire NHS Foundation Trust Public Inquiry. Available at:

http://www.midstaffspublicinquiry.com/report (accessed on 04.11.2014).

1. Culture of Care Barometer Kings College London. Available at:

http://www.kcl.ac.uk/nursing/research/ (accessed on 04.11.2014).

1. Association for Video Interaction Guidance UK Available at:
https://www.dropbox.com/sh/rcn8sbkytyayrxy/PsN30yl1p5 (accessed 4.11.2014).

1. Kennedy, H, Landor, M and Todd, L (eds). Video Interaction Guidance: a

relationship-based Intervention to Promote Attunement, Empathy and Wellbeing. (2011)

Jessica Kingsley Publishers.

1. Kennedy H, Landor, M. and Todd L (eds). Kennedy, $H$. Video Enhanced Reflective

Practice: Professional Development through Attuned Interaction London: (2015) Jessica Kingsley.

1. Hellier C. Supporting professional and organisational development to 'reflect'and 're-view' in post-school work. In Kennedy $\mathrm{H}$, et al Video Enhanced Reflective Practice: Professional

Development through Attuned Interaction London: (2015) Jessica Kingsley.

1. Greene A. Cartwright, E. \& Webster, C. 'Integrating VERP into Medical Education, In Video Enhanced Reflective Practice - professional development through attuned interactions' (In Press April 2015).

2. Perth Royal Infirmary. Care of older people Inspection Report September 2014. Available at: http://www.healthcareimprovementscotland.org/system_pag es/published_resources_search. $a s p x ? f=6: 317 \& q=$ perth (accessed on 04/11/2014).

\section{Declaration of interests}

Nothing to declare. 